\title{
Constructions and bounds for separating hash families
}

\author{
X. Niu and H. Cao * \\ Institute of Mathematics, \\ Nanjing Normal University, Nanjing 210023, China
}

\begin{abstract}
In this paper, we present a new construction for strong separating hash families by using hypergraphs and obtain some optimal separating hash families. We also improve some previously known bounds of separating hash families.
\end{abstract}

Key words: Hash family, separating hash family, strong separating hash family, hypergraph

\section{Introduction}

Let $X$ and $Y$ be two finite sets of sizes $n$ and $m$ respectively. An $(N ; n, m)$-hash family $\mathcal{F}$ is a family of functions from $X$ to $Y$ with $|\mathcal{F}|=N$. For all pairwise disjoint subsets $C_{1}, C_{2}, \ldots, C_{t} \subseteq X$, if there exists some $f$ such that $f\left(C_{i}\right) \cap f\left(C_{j}\right)=\emptyset$ for all $1 \leq i<j \leq t$. Then $C_{1}, C_{2}, \ldots, C_{t}$ are separable in $\mathcal{F}$, and the function $f$ is said to separate the sets $C_{1}, C_{2}, \ldots, C_{t}$.

Given positive integers $w_{1}, w_{2}, \ldots, w_{t}$, we say $\mathcal{F}$ is a $\left\{w_{1}, w_{2}, \ldots, w_{t}\right\}$-separating hash family, denoted by $\operatorname{SHF}\left(N ; n, m,\left\{w_{1}, w_{2}, \ldots, w_{t}\right\}\right)$, if for all pairwise disjoint subsets $C_{1}, C_{2}, \ldots, C_{t} \subseteq X$ with $\left|C_{i}\right|=w_{i}$ for $i=1,2, \ldots, t$, there exists some $f \in \mathcal{F}$ which separates $C_{1}, C_{2}, \ldots, C_{t}$. The parameter multiset $\left\{w_{1}, w_{2}, \ldots, w_{t}\right\}$ is called the type of $\mathcal{F}$. For the sake of brevity, we use SHF to denote separating hash family, and we also use $\left\{w_{1}^{q_{1}}, w_{2}^{q_{2}}, \ldots, w_{t}^{q_{t}}\right\}$ to denote the multiset in which there are exactly $q_{i}$ copies of $w_{i}$ and $w_{i}<w_{j}$ for $1 \leq i<j \leq t$. Further, $w^{1}$ will be written as $w$. $\operatorname{An} \operatorname{SHF}\left(N ; n, m,\left\{1^{q}, w\right\}\right)$ with $w \geq 2$ is also called a strong separating hash family.

Separating hash families were first introduced by Stinson, Trung and Wei [26]. It can be used to construct frameproof codes, secure frameproof codes and parent-identifying codes, see [7, 22, 25, 26]. Most results of the known papers on separating hash families are focused on their bounds and constructions, see $[1-6,10-12,12,14,15,24,27,28]$.

Given an $\operatorname{SHF}\left(N ; n, m,\left\{w_{1}, w_{2}, \ldots, w_{t}\right\}\right)$, we construct an $N \times n$ matrix $A=\left(a_{i, j}\right)$ having entries on a set of $m$ elements such that $a_{i, j}=f_{i}\left(x_{j}\right)$ where $f_{1}, f_{2}, \ldots, f_{N}$ are some fixed ordering of the functions in $\mathcal{F}$ and $x_{1}, \ldots, x_{n}$ are elements of $X$. This matrix is called the representation matrix of

\footnotetext{
${ }^{*}$ Research supported by the National Natural Science Foundation of China under Grant No. 11571179, and the Priority Academic Program Development of Jiangsu Higher Education Institution.
} 
the SHF. For all disjoint sets of columns $C_{1}, C_{2}, \ldots, C_{t}$ of $A$ with $\left|C_{i}\right|=w_{i}, 1 \leq i \leq t$, there exists at least one row $r$ of $A$ such that $\left\{a_{r, x}: x \in C_{i}\right\} \cap\left\{a_{r, y}: y \in C_{j}\right\}=\emptyset$ holds for all $1 \leq i<j \leq t$. We say the row $r$ separates the sets $C_{1}, C_{2}, \ldots, C_{t}$. An $\operatorname{SHF}\left(N ; n, m,\left\{w_{1}, w_{2}, \cdots, w_{t}\right\}\right)$ is called optimal if $n$ is maximum for given $N, m, w_{1}, w_{2}, \cdots, w_{t}$ or if $N$ is minimum for given $n, m, w_{1}, w_{2}, \cdots, w_{t}$.

In the literature optimal results for separating hash families are quite rare. In this paper, we present a new construction for strong separating hash families by using hypergraphs and obtain some optimal separating hash families. It is easy to see that a separating hash family with type $\left\{w_{1}, w_{2}, \ldots, w_{t}\right\}$ is a separating hash family with type $\left\{w_{1}+w_{2}, w_{3}, \ldots, w_{t}\right\}$ and also a separating hash family with type $\left\{w, w_{2}, \ldots, w_{t}\right\}$ with $w \leq w_{1}$. So separating hash families with type $\left\{w_{1}, w_{2}, \ldots, w_{t}\right\}$ can yield separating hash families with type $\left\{w_{1}^{\prime}, w_{2}^{\prime}\right\}$. Thus it is valuable to study the bound of separating hash families with type $\left\{w_{1}, w_{2}\right\}$.

This paper is organized as follows. In the next section, we present a new construction for strong separating hash families by using hypergraphs and present some optimal strong separating hash families. In Section 3 we construct an optimal $\operatorname{SHF}(4 ; 10,4,\{2,2\})$ and use it to improve the known bound for an $\operatorname{SHF}(2 w ; n, m,\{w, w\})$. In the last section, we improve the known bound for an $\operatorname{SHF}\left(w_{1}+w_{2} ; n, m,\left\{w_{1}, w_{2}\right\}\right)$ and use it to improve the known bound for an $\operatorname{SHF}\left(\sum_{i=1}^{t} w_{i} ; n, m,\left\{w_{1}, w_{2}, \ldots, w_{t}\right\}\right)$.

\section{A new construction for strong separating hash family}

In this section, we will give a construction for strong separating hash families by using hypergraphs, and we also give some tight results for strong separating hash families. A hypergraph is a pair $H=(V, E)$, where $V$ is a finite set whose elements are called vertices and $E$ is a family of subsets of $V$, called edges. It is $k$-uniform if each of its edges contains precisely $k$ vertices.

Theorem 2.1 Let $V=\left\{x_{1}, x_{2}, \ldots, x_{n}\right\}$ and $E=\left\{B_{1}, B_{2}, \ldots, B_{N}\right\}$, where $B_{i}$ is an m-subset of $V, 1 \leq i \leq N$. If $G=(V, E)$ is an $m$-uniform hypergraph with the property that any $l$ vertices are contained in exactly one edge, then there exists an $\operatorname{SHF}\left(N ; n, m+1,\left\{1^{w_{1}}, w_{2}\right\}\right)$ for all positive integers $w_{1}$ and $w_{2}$ satisfying $w_{1} \leq l$ and $w_{1}+w_{2} \leq n$.

Proof: Let $B_{i}=\left\{y_{i, 1}, y_{i, 2}, \ldots, y_{i, m}\right\}, 1 \leq i \leq N$. Define an $N \times n$ matrix $A=\left(a_{i, j}\right)$ by

$$
a_{i, j}= \begin{cases}b, & \text { if } x_{j}=y_{i, b} \\ 0, & \text { otherwise. }\end{cases}
$$

Now we prove $A$ is a representation matrix of an $\operatorname{SHF}\left(N ; n, m+1,\left\{1^{w_{1}}, w_{2}\right\}\right)$. Let $\mathcal{C}=\left\{c_{1}, c_{2}, \ldots, c_{n}\right\}$ denote the column set of $A$. Let $C_{1}, C_{2}, \ldots, C_{w_{1}}, C_{w_{1}+1}$ be pairwise disjoint subsets of $\mathcal{C}$ such that $C_{i}=\left\{c_{s_{i}}\right\}$ for $i=1,2, \ldots, w_{1}$ and $\left|C_{w_{1}+1}\right|=w_{2} \leq n-w_{1}$. Then $\left|C_{1} \cup C_{2} \cup \cdots \cup C_{w_{1}}\right|=w_{1} \leq l$, $C_{w_{1}+1} \subset \mathcal{C} \backslash\left\{c_{s_{1}}, c_{s_{2}}, \ldots, c_{s_{w_{1}}}\right\}$.

Since $G=(V, E)$ is an $m$-uniform hypergraph with the property that any $l$ vertices are contained in exactly one edge, we can find an edge $B_{t} \in E$ such that $\left\{x_{s_{1}}, x_{s_{2}}, \ldots, x_{s_{w_{1}}}\right\} \subset B_{t}$. Let $B_{t}=$ 
$\left\{x_{s_{1}}, x_{s_{2}}, \ldots, x_{s_{w_{1}}}, x_{s_{w_{1}+1}}, \ldots, x_{s_{m}}\right\}$. Then $\left\{a_{t, s_{1}}, a_{t, s_{2}}, \ldots, a_{t, s_{m}}\right\}=\{1,2, \ldots, m\}$ and $a_{t, j}=0$ for any $j \in\{1,2, \ldots, n\} \backslash\left\{s_{1}, s_{2}, \ldots, s_{m}\right\}$. Thus, $\left\{a_{t, k}: c_{k} \in C_{i}\right\} \cap\left\{a_{t, k}: c_{k} \in C_{j}\right\}=\emptyset$ holds for all $1 \leq i<j \leq w_{1}+1$. Therefore, the $t$-th row of $A$ can separate $C_{1}, C_{2}, \ldots, C_{w_{1}+1}$.

Example 2.2 Let $V=\mathbb{Z}_{7}$ and $E=\left\{\{i, i+1, i+3\}: i \in \mathbb{Z}_{7}\right\}$. We can obtain the following representation matrix of an $\operatorname{SHF}\left(7 ; 7,4,\left\{1^{2}, 5\right\}\right)$ by Theorem 2.1.

\begin{tabular}{|l|l|l|l|l|l|l|}
\hline 1 & 2 & 0 & 3 & 0 & 0 & 0 \\
\hline 0 & 1 & 2 & 0 & 3 & 0 & 0 \\
\hline 0 & 0 & 1 & 2 & 0 & 3 & 0 \\
\hline 0 & 0 & 0 & 1 & 2 & 0 & 3 \\
\hline 3 & 0 & 0 & 0 & 1 & 2 & 0 \\
\hline 0 & 3 & 0 & 0 & 0 & 1 & 2 \\
\hline 2 & 0 & 3 & 0 & 0 & 0 & 1 \\
\hline
\end{tabular}

For our results, we need the following conclusion on $m$-uniform hypergraphs.

Theorem 2.3 ([16 18]) 1. For any $3 \leq m \leq 5$, there exists an $m$-uniform hypergraph $G=(V, E)$ with the property that any two vertices are contained in exactly one edge, where $|V|=n$ satisfying $n \equiv 1, m\left(\bmod m^{2}-m\right)$, and $|E|=\frac{n(n-1)}{m(m-1)}$.

2. For any $n \equiv 2,4(\bmod 6)$, there exists a 4 -uniform hypergraph with $n$ vertices and $\frac{n(n-1)(n-2)}{24}$ edges such that any three vertices are contained in exactly one edge.

3. For any prime power $m$ and integer $l \geq 2$, there exists an $(m+1)$-uniform hypergraph $G=(V, E)$ with the property that any three vertices are contained in exactly one edge, where $|V|=n$ satisfying $n=m^{l}+1$, and $|E|=\frac{\left(\begin{array}{c}n \\ 3\end{array}\right)}{\left(\begin{array}{c}m+1 \\ 3\end{array}\right)}$.

By Theorems 2.1 and 2.3 we have the following theorem.

Theorem 2.4 1. Let $3 \leq m \leq 5, n \equiv 1, m\left(\bmod m^{2}-m\right), n>m$, and $N=\frac{n(n-1)}{m(m-1)}$. Then there is an $\operatorname{SHF}\left(N ; n, m+1,\left\{1^{2}, n-2\right\}\right)$.

2. Let $n \equiv 2,4(\bmod 6), n \geq 8$ and $N=\frac{n(n-1)(n-2)}{24}$. Then there is an $\operatorname{SHF}\left(N ; n, 5,\left\{1^{3}, n-3\right\}\right)$.

3. Let $n=m^{l}+1$, where $m$ is a prime power and $l \geq 2$. Let $N=\frac{\left(\begin{array}{c}n \\ 3\end{array}\right)}{\left(\begin{array}{c}m+1 \\ 3\end{array}\right)}$. Then there is an $\operatorname{SHF}\left(N ; n, m+2,\left\{1^{3}, n-3\right\}\right)$.

Now we have obtained some new strong separating hash families from Theorem 2.4. We continue to show that these results from Theorem 2.4 are all tight by discussing the lower bound of $N$ for an $\operatorname{SHF}\left(N ; w_{1}+w_{2}, m,\left\{1^{w_{1}}, w_{2}\right\}\right)$. When $w_{1}+w_{2} \leq m$, it is easy to see that $N \geq 1$. So this case is trivial and we only need to deal with the case $w_{1}+w_{2}>m$. 
Theorem 2.5 If there is an $\operatorname{SHF}\left(N ; w_{1}+w_{2}, m,\left\{1^{w_{1}}, w_{2}\right\}\right)$ with $w_{1}+w_{2}>m$, then $N \geq \frac{\left(\begin{array}{c}w_{1}+w_{2} \\ w_{1}\end{array}\right)}{\left(\begin{array}{c}m-1 \\ w_{1}\end{array}\right)}$.

Proof: Let $A=\left(a_{i, j}\right)$ be a representation matrix of an $\operatorname{SHF}\left(N ; w_{1}+w_{2}, m,\left\{1^{w_{1}}, w_{2}\right\}\right)$ with the column set $\mathcal{C}=\left\{c_{1}, c_{2}, \ldots, c_{n}\right\}$. Then the total number of the pairwise disjoint subsets of $\mathcal{C}$ which need to be separated is $\left(\begin{array}{c}w_{1}+w_{2} \\ w_{1}\end{array}\right)$. On the other hand, suppose $C_{1}, C_{2}, \ldots, C_{w_{1}+1}$ are pairwise disjoint subsets of $\mathcal{C}$ such that $\left|C_{w_{1}+1}\right|=w_{2}$ and $\left|C_{j}\right|=1$ for $1 \leq j \leq w_{1}$. Let $C_{j}=\left\{c_{i_{j}}\right\}$ for $1 \leq j \leq w_{1}$. If these $w_{1}+1$ subsets can be separated by the $i$ th row, then the element $a_{i, j}$ appears exactly once in the $i$ th row for any $j \in\left\{i_{1}, i_{2}, \ldots, i_{w_{1}}\right\}$. Since $w_{1}+w_{2}>m$, there are at most $m-1$ elements occurring exactly once in each row. So the maximum number of the pairwise disjoint subsets which can be separated by each row is $\left(\begin{array}{c}m-1 \\ w_{1}\end{array}\right)$. Thus, $N \geq \frac{\left(\begin{array}{c}w_{1}+w_{2} \\ w_{1}\end{array}\right)}{\left(\begin{array}{c}m-1 \\ w_{1}\end{array}\right)}$.

By Theorem 2.5, it is easy to check that these strong separating hash families constructed in Theorem 2.4 are all optimal. For more results on $m$-uniform hypergraphs with the property that any $t$ vertices are contained in exactly one edge, see [8] (pages 72-73, 82-84, 661). For example, there are a 5-uniform hypergraph with 11 vertices and 66 edges such that any four vertices are contained in exactly one edge, and a 6-uniform hypergraph with 12 vertices and 132 edges such that any five vertices are contained in exactly one edge, see [8] (page 661). By Theorems 2.1 and 2.5. we obtain an optimal $\operatorname{SHF}\left(66 ; 11,5,\left\{1^{4}, 7\right\}\right)$ and an optimal $\operatorname{SHF}\left(132 ; 12,6,\left\{1^{5}, 7\right\}\right)$.

Remark 1 : For the bounds of strong separating hash families, Sarkar and Stinson [22] proved that there exists an infinite class of $\operatorname{SHF}\left(N ; n, m,\left\{1^{w_{1}}, w_{2}\right\}\right)$ for which $N$ is $O\left(\left(w_{1}\left(w_{1}+w_{2}\right)\right)^{\log { }^{*} n} \log n\right)$. Liu and Shen [20] gave an infinite constructions of the $\operatorname{SHF}\left(N ; n, m,\left\{1^{w_{1}}, w_{2}\right\}\right)$ for which $N$ is $O(\log n)$. Guo and Stinson [15] proved $N \geq\left(\begin{array}{c}n \\ m-1\end{array}\right)$ when $w_{1} \geq m-1$ and $w_{1}+w_{2} \leq n \leq$ $2\left(w_{1}+w_{2}\right)-m$. Now we compare our conclusion with the bound in [15]. By the definition of an SHF, it is obvious that $m-1 \geq w_{1}$. So Guo and Stinson's bound in [15] can be restated as $N \geq\left(\begin{array}{c}n \\ w_{1}\end{array}\right)$ when $m-1=w_{1}$ and $w_{1}+w_{2} \leq n \leq w_{1}+2 w_{2}-1$. When $n=w_{1}+w_{2}$ and $m-1=w_{1}$, it is easy to see that we have the same conclusion. But for the case $n=w_{1}+w_{2}$ and $m-1>w_{1}$, we have a new tight bound.

Actually, the hypergraphs used in Theorem 2.1 need not to be $k$-uniform, and any $l$ vertices need not to be contained in exactly one edge. We can generalize this construction to a hypergraph with different edge sizes. The proof of the following theorem is similar to that of Theorem 2.1, So we just present the theorem without proof.

Theorem 2.6 Suppose there is a hypergraph with $n$ vertices and $N$ edges such that the maximum edge size is $m$ and any $l$ vertices are contained in at least one edge, then there exists an $\operatorname{SHF}\left(N ; n, m+1,\left\{1^{w_{1}}, w_{2}\right\}\right)$ for all positive integers $w_{1}$ and $w_{2}$ satisfying $w_{1} \leq l$ and $w_{1}+w_{2} \leq n$.

Below are some results of $k$-uniform hypergraphs such that any $l$ vertices are contained in at least one edge.

Theorem $2.7([8,[9])$ 1. There exists an 3-uniform hypergraph $G=(V, E)$ with the property that 
any two vertices are contained in at least one edge, where $|V|=n \geq 5$ and $|E|=\left\lceil\frac{n}{3}\left\lceil\frac{n-1}{2}\right\rceil\right\rceil$.

2.There exists an 4-uniform hypergraph $G=(V, E)$ with the property that any two vertices are contained in at least one edge, where $|V|=n \geq 6$ and $|E|=\left\lceil\frac{n}{4}\left\lceil\frac{n-1}{3}\right\rceil\right\rceil+c$, where $c=1$ if $n=7,9,10 ; c=2$ if $n=19$; and $c=0$ for all other values of $n$.

3. There exists an 4-uniform hypergraph $G=(V, E)$ with the property that any three vertices are contained in at least one edge, where $|V|=n$ and $|E|=\left\lceil\frac{n}{4}\left\lceil\frac{n-1}{3}\left\lceil\frac{n-2}{2}\right\rceil\right\rceil\right\rceil$ satisfying $n \neq 12 t+7$ for any $t \in([0,12] \backslash\{6\}) \cup\{16,21,23,25,29\}$.

For more results on $m$-uniform hypergraphs with the property that any $t$ vertices are contained in at least one edge, see [8] (pages 366-372). By Theorems 2.6] and 2.7 we have the following theorem.

Theorem 2.8 1. For $n \geq 5$, there is an $\operatorname{SHF}\left(N ; n, 4,\left\{1^{2}, n-2\right\}\right)$ with $N=\left\lceil\frac{n}{3}\left\lceil\frac{n-1}{2}\right\rceil\right\rceil$.

2. For $n=7,9,10$, there is an $\operatorname{SHF}\left(N ; n, 5,\left\{1^{2}, n-2\right\}\right)$ with $N=\left\lceil\frac{n}{4}\left\lceil\frac{n-1}{3}\right\rceil\right\rceil+1$.

3. There is an $\operatorname{SHF}\left(31 ; 19,5,\left\{1^{2}, 17\right\}\right)$.

4. For $n \geq 6$ and $n \neq 7,9,10,19$, there is an $\operatorname{SHF}\left(N ; n, 5,\left\{1^{2}, n-2\right\}\right)$ with $N=\left\lceil\frac{n}{4}\left\lceil\frac{n-1}{3}\right\rceil\right\rceil$.

5. For any $n \neq 12 t+7, t \in([0,12] \backslash\{6\}) \cup\{16,21,23,25,29\}$, there is an $\operatorname{SHF}\left(N ; n, 5,\left\{1^{3}, n-3\right\}\right)$ with $N=\left\lceil\frac{n}{4}\left\lceil\frac{n-1}{3}\left\lceil\frac{n-2}{2}\right\rceil\right\rceil\right\rceil$.

By Theorem 2.6, we can obtain more results on strong separating hash families by using the known results on hypergraphs. For example, there exists a hypergraph with 10 vertices and 12 edges with size 3 or 4 such that any two vertices are contained in exactly one edge, see [8] (page 231). Thus, we can construct an $\operatorname{SHF}\left(12 ; 10,5,\left\{1^{2}, 8\right\}\right)$. There exists a hypergraph with 16 vertices and 68 edges with size 4 or 5 such that any three vertices are contained in exactly one edge, see [8] (page 662). Thus, we can construct an $\operatorname{SHF}\left(68 ; 16,6,\left\{1^{3}, 13\right\}\right)$. There exists a hypergraph with 17 vertices and 252 edges with size 5 or 6 such that any four vertices are contained in exactly one edge, see [8](page 663). Thus, we can construct an $\operatorname{SHF}\left(252 ; 17,7,\left\{1^{4}, 13\right\}\right)$. There also exists a hypergraph with 16 vertices and 478 edges with size 6 or 8 such that any five vertices are contained in exactly one edge, see [8] (page 661). Thus, we can construct an $\operatorname{SHF}\left(478 ; 16,9,\left\{1^{5}, 11\right\}\right)$. For more results of the known papers on hypergraphs, see [8].

For given parameters $n, m, w_{1}, w_{2}$ of an $\operatorname{SHF}\left(N ; n, m+1,\left\{1^{w_{1}}, w_{2}\right\}\right)$, we can also obtain the following bounds on $N$. Let $M(n, k, l)$ denote the minimum possible number of edges of a $k$ uniform hypergraph with the property that any $l$ vertices are contained in at least one edge.

Theorem 2.9 ([21] $) \frac{\left(\begin{array}{l}n \\ l\end{array}\right)}{\left(\begin{array}{c}k \\ l\end{array}\right)} \leq M(n, k, l) \leq(1+o(1)) \frac{\left(\begin{array}{c}n \\ l\end{array}\right)}{\left(\begin{array}{c}k \\ l\end{array}\right)}$, where the $o(1)$ term tends to zero as $n$ tends to infinity.

According to Theorems 2.6 and 2.9, we have the following theorem. 
Theorem 2.10 If there exists an $\operatorname{SHF}\left(N ; n, m+1,\left\{1^{w_{1}}, w_{2}\right\}\right)$ with $w_{1} \leq m$, then $N \leq(1+$ $o(1)) \frac{\left(\begin{array}{c}n \\ w_{1}\end{array}\right)}{\left(\begin{array}{c}m \\ w_{1}\end{array}\right)}$. Furthermore, if $n=w_{1}+w_{2}$, we have $\frac{\left(\begin{array}{c}n \\ w_{1}\end{array}\right)}{\left(\begin{array}{c}m \\ w_{1}\end{array}\right)} \leq N \leq(1+o(1)) \frac{\left(\begin{array}{c}n \\ w_{1}\end{array}\right)}{\left(\begin{array}{c}m \\ w_{1}\end{array}\right)}$.

\section{$3 \quad$ An improved bound for $\operatorname{SHF}(2 w ; n, m,\{w, w\})$}

In this section, we shall give a new bound for an $\operatorname{SHF}(2 w ; n, m,\{w, w\})$ with $w \geq 2$. This bound is useful in the next section. We start with some definitions and notations.

Let $A$ and $B$ be two matrices. If $B$ can be obtained from $A$ by permuting the rows and/or columns and/or elements, then we say that $A$ is isomorphic to $B$.

Lemma 3.1 If $A$ is a representation matrix of an $\operatorname{SHF}\left(N ; n, m,\left\{w_{1}, w_{2}, \ldots, w_{t}\right\}\right)$, and if $B$ is isomorphic to $A$, then $B$ is also a representation matrix of an $\operatorname{SHF}\left(N ; n, m,\left\{w_{1}, w_{2}, \ldots, w_{t}\right\}\right)$.

In order to find good upper bounds for SHFs, we often use one of the general methods to show that a particular choice of $n$ implies that the representation matrix $A$ always contains a submatrix which is impossible in an SHF with given parameters. Such a submatrix is referred to a forbidden configuration.

Lemma 3.2 If $A$ is a representation matrix of an $\operatorname{SHF}(4 ; n, m,\{2,2\})$, then any submatrix of $A$ can't be isomorphic to the following forbidden configurations $F_{1}, F_{2}$ and $F_{3}$.

\begin{tabular}{|c|c|c|c|}
\multicolumn{4}{c}{$F_{1}$} \\
\hline$a$ & $a$ & $*$ & $*$ \\
\hline$b$ & $b$ & $*$ & $*$ \\
\hline$*$ & $*$ & $c$ & $c$ \\
\hline$*$ & $*$ & $d$ & $d$ \\
\hline
\end{tabular}

\begin{tabular}{|c|c|c|c|}
\multicolumn{4}{c}{$F_{2}$} \\
\hline$a$ & $a$ & $*$ & $*$ \\
\hline$b$ & $b$ & $*$ & $*$ \\
\hline$x$ & $*$ & $x$ & $*$ \\
\hline$*$ & $v$ & $*$ & $v$ \\
\hline
\end{tabular}

\begin{tabular}{|c|c|c|c|}
\multicolumn{4}{c}{$F_{3}$} \\
\hline$a$ & $a$ & $*$ & $*$ \\
\hline$*$ & $b$ & $b$ & $*$ \\
\hline$x$ & $*$ & $*$ & $x$ \\
\hline$*$ & $*$ & $v$ & $v$ \\
\hline
\end{tabular}

Proof: It is easy to check that in $F_{1}$ or $F_{3}$ the column sets $C_{1}=\{1,3\}$ and $C_{2}=\{2,4\}$ are not separable, and in $F_{2}$ the column sets $C_{1}=\{1,4\}$ and $C_{2}=\{2,3\}$ are not separable.

Suppose $A=\left(a_{i, j}\right)$ is an $N \times n$ representation matrix of an SHF on $m$ elements in $Y$. We need the following notations for the following lemmas. Let

$$
\begin{gathered}
\lambda_{x}^{i}=\left|\left\{j: a_{i, j}=x, 1 \leq j \leq n\right\}\right|, 1 \leq i \leq N, x \in Y, \\
\lambda_{\max }=\max \left\{\lambda_{x}^{i}: 1 \leq i \leq N, x \in Y\right\}, \text { and } \\
d_{i, j}(x, y)=\left|\left\{k: a_{i, k}=x, a_{j, k}=y, 1 \leq k \leq n\right\}\right|, 1 \leq i<j \leq N .
\end{gathered}
$$

Suppose $B$ is a submatrix of $A$, we shall use $A-B$ to denote the matrix obtained by removing all these entries in $B$ from $A$. 
Lemma 3.3 Suppose $A$ is a representation matrix of an $\operatorname{SHF}(4 ; n, m,\{2,2\})$ with $m \geq 3$. If there is a pair of elements $x$ and $y$ such that $d_{i, j}(x, y) \geq 2$, then $n \leq(m-1)^{2}+1$.

Proof: Without loss of generality, we may assume $d_{1,2}(a, b) \geq 2$. Then the matrix $B$ in Table 1 is a submatrix of $A$.

\section{Table 1: Three submatrices of $A$}

$$
B=\begin{array}{|l|l|}
\hline a & a \\
\hline b & b \\
\hline x & y \\
\hline u & v \\
\hline
\end{array}
$$

$$
E=\begin{array}{|c|c|c|}
\hline a & a & * \\
\hline b & b & * \\
\hline x & y & y \\
\hline u & v & u \\
\hline
\end{array}
$$$$
M=\begin{array}{|c|c|c|c|}
\hline a & a & * & * \\
\hline b & b & * & * \\
\hline x & y & y & x \\
\hline u & v & u & v \\
\hline
\end{array}
$$

By Lemma 3.1 we only need to consider the following 5 cases.

1. $\lambda_{x}^{3}=\lambda_{y}^{3}=1$. Then $A-B$ is a representation matrix of an $\operatorname{SHF}(4 ; n-2, m,\{2,2\})$, and there are at most $m-2$ distinct elements in row three. By the pigeonhole principle, there is an element $t$ in row four such that $\lambda_{t}^{4} \geq\left\lceil\frac{n-2}{m}\right\rceil$. If $\lambda_{t}^{4}>m-2$, then we have an element $g$ in row three such that $d_{3,4}(g, t) \geq 2$ by the pigeonhole principle. So we obtain a submatrix of $A$ which is isomorphic to the forbidden configuration $F_{1}$. Thus, $\lambda_{t}^{4} \leq m-2$. Then we have $\left\lceil\frac{n-2}{m}\right\rceil \leq m-2$. So, $n \leq m^{2}-2 m+2$.

2. $\lambda_{x}^{3}=1, \lambda_{y}^{3}>1, \lambda_{u}^{4}=1, \lambda_{v}^{4}>1$. Let $D$ be the $4 \times(n-1)$ matrix obtained from $A$ by removing column one. Then $D$ is a representation matrix of an $\operatorname{SHF}(4 ; n-1, m,\{2,2\})$, and there are at most $m-1$ distinct elements in the last two rows. By the pigeon hole principle, there is an element $t$ such that $\lambda_{t}^{4} \geq\left\lceil\frac{n-1}{m-1}\right\rceil$. Then we have $\left\lceil\frac{n-1}{m-1}\right\rceil \leq m-1$ and $n \leq m^{2}-2 m+2$.

3. $\lambda_{x}^{3}=1, \lambda_{y}^{3}>1, \lambda_{u}^{4}>1, \lambda_{v}^{4}=1$. By Lemma 3.2( forbidden configuration $F_{2}$ ) we know that $\lambda_{u}^{4}=\lambda_{y}^{3}=2$, and $A-E$ is a representation matrix of an $\operatorname{SHF}(4 ; n-3, m,\{2,2\})$, and there are at most $m-2$ distinct elements in rows three and four. Similarly we have $\left\lceil\frac{n-3}{m-2}\right\rceil \leq m-2$ and $n \leq m^{2}-4 m+7$.

4. $\lambda_{x}^{3}=1, \lambda_{y}^{3}>1, \lambda_{u}^{4}>1, \lambda_{v}^{4}>1$. In this case, it also holds that $\lambda_{u}^{4}=\lambda_{y}^{3}=2$ by Lemma 3.2 (forbidden configuration $F_{2}$ ), and $A-E$ is a representation matrix of an $\mathrm{SHF}(4 ; n-$ $3, m,\{2,2\})$, and there are at most $m-2$ and $m-1$ distinct elements in rows three and four respectively. Thus we have $\left\lceil\frac{n-3}{m-2}\right\rceil \leq m-1$ and $n \leq m^{2}-3 m+5$.

5. $\lambda_{x}^{3}>1, \lambda_{y}^{3}>1, \lambda_{u}^{4}>1, \lambda_{v}^{4}>1$. By Lemma 3.2 (forbidden configuration $F_{2}$ ) we know that $\lambda_{x}^{3}=\lambda_{y}^{3}=\lambda_{u}^{4}=\lambda_{v}^{4}=2$, and $A-M$ is a representation matrix of an $\operatorname{SHF}(4 ; n-4, m,\{2,2\})$, and there are at most $m-2$ distinct elements in the last two rows. So we have $\left\lceil\frac{n-4}{m-2}\right\rceil \leq m-2$ and $n \leq m^{2}-4 m+8$.

Combining the above 5 cases with the condition $m \geq 3$, we have obtained $n \leq(m-1)^{2}+1$. The proof is complete.

Now we prove that if there exists an $\operatorname{SHF}(4 ; n, 4,\{2,2\})$, then $n \leq 10$. To prove this conclusion, we assume that an $\operatorname{SHF}(4 ; 11,4,\{2,2\})$ exists and we get a contradiction. 
Lemma 3.4 If $A$ is a representation matrix of an $\operatorname{SHF}(4 ; 11,4,\{2,2\})$, then $3 \leq \lambda_{\text {max }} \leq 4$, $d_{i, j}(x, y) \leq 1$ for all admissible elements $x, y$ and parameters $i, j$, and each row of $A$ is isomorphic to $R_{1}$ or $R_{2}$ as below.
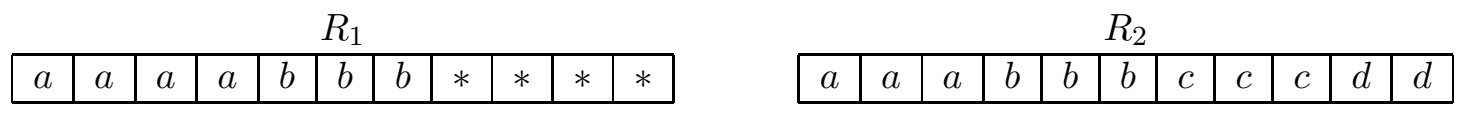

Proof: By Lemma 3.3, $d_{i, j}(x, y) \leq 1$ holds for any admissible elements $x, y$ and parameters $i, j$. Then it is obvious that $\lambda_{\max } \leq 4$. Also we know that $\lambda_{\max } \geq 3$ since $4 \times 2=8<11$. So we have $3 \leq \lambda_{\max } \leq 4$, and it's easy to see that each row of $A$ is isomorphic to $R_{1}$ or $R_{2}$.

Lemma 3.5 If $A$ is a representation matrix of an $\operatorname{SHF}(4 ; 11,4,\{2,2\})$, then there is at most one row of $A$ which is isomorphic to $R_{1}$.

Proof: Assume, by contradiction, that $A$ has two rows which are both isomorphic to $R_{1}$. Without loss of generality, we assume both of the first two rows are isomorphic to $R_{1}$ and $\lambda_{a}^{1}=4$. By Lemma 3.3, $d_{1,2}(a, g)=1$ holds for any element $g$ which is in the second row and in the same column with $a$. Since there are four distinct elements in total in the second row, there exists an element $e$ such that $d_{1,2}(a, e)=1$ and $\lambda_{e}^{2}=4$. So we may assume the first 7 columns of $A$ is the submatrix as below.

\begin{tabular}{|c|c|c|c|c|c|c|}
\hline$a$ & $a$ & $a$ & $a$ & $*$ & $*$ & $*$ \\
\hline$*$ & $*$ & $*$ & $e$ & $e$ & $e$ & $e$ \\
\hline$x$ & $y$ & $z$ & $t$ & $x$ & $y$ & $z$ \\
\hline$u$ & $v$ & $i$ & $j$ & $a_{4,5}$ & $a_{4,6}$ & $a_{4,7}$ \\
\hline
\end{tabular}

Then by Lemma 3.4 we have $\left(a_{4,5}, a_{4,6}, a_{4,7}\right)=(v, i, u)$ or $(i, u, v)$. We distinguish two cases.

1. $\left(a_{4,5}, a_{4,6}, a_{4,7}\right)=(v, i, u)$. Then we have the following submatrix.

\begin{tabular}{|c|c|c|c|c|c|c|}
\hline$a$ & $a$ & $a$ & $a$ & $*$ & $*$ & $*$ \\
\hline$*$ & $*$ & $c$ & $e$ & $e$ & $e$ & $e$ \\
\hline$x$ & $y$ & $z$ & $t$ & $x$ & $y$ & $z$ \\
\hline$u$ & $v$ & $i$ & $j$ & $v$ & $i$ & $u$ \\
\hline
\end{tabular}

Thus, the column sets $C_{1}=\{1,6\}$ and $C_{2}=\{3,5\}$ are not separable, a contradiction.

2. $\left(a_{4,5}, a_{4,6}, a_{4,7}\right)=(i, u, v)$. Then we also have the following submatrix.

\begin{tabular}{|c|c|c|c|c|c|c|}
\hline$a$ & $a$ & $a$ & $a$ & $*$ & $*$ & $*$ \\
\hline$*$ & $*$ & $c$ & $e$ & $e$ & $e$ & $e$ \\
\hline$x$ & $y$ & $z$ & $t$ & $x$ & $y$ & $z$ \\
\hline$u$ & $v$ & $i$ & $j$ & $i$ & $u$ & $v$ \\
\hline
\end{tabular}

Thus, the column sets $C_{1}=\{1,7\}$ and $C_{2}=\{2,5\}$ are not separable, a contradiction. 
Lemma 3.6 If $A$ is a representation matrix of an $\operatorname{SHF}(4 ; 11,4,\{2,2\})$, then each row of $A$ is isomorphic to $R_{2}$.

Proof: By Lemma 3.5, we know that $A$ has no two rows which are both isomorphic to $R_{1}$. So we assume that the first row of $A$ is isomorphic to $R_{1}$ and the other rows of $A$ are isomorphic to $R_{2}$. Without loss of generality, we start with the following submatrix.

\begin{tabular}{|c|c|c|c|c|c|c|c|c|c|c|}
\hline$a$ & $a$ & $a$ & $a$ & $*$ & $*$ & $*$ & $*$ & $*$ & $*$ & $*$ \\
\hline$b$ & $c$ & $d$ & $e$ & $b$ & $b$ & $c$ & $c$ & $d$ & $d$ & $e$ \\
\hline$x$ & $y$ & $z$ & $t$ & $*$ & $*$ & $*$ & $*$ & $*$ & $*$ & $x$ \\
\hline$u$ & $v$ & $i$ & $j$ & $*$ & $*$ & $*$ & $*$ & $*$ & $*$ & $*$ \\
\hline
\end{tabular}

By Lemma 3.4, $a_{4,11}=v$ or $i$. By Lemma 3.1, we only consider $a_{4,11}=v$. By Lemma 3.2(forbidden configuration $F_{3}$ ), considering the column set $\{3,4,11\}$, we have

$$
d_{3,4}(z, v)=d_{3,4}(x, i)=0
$$

1. $\lambda_{x}^{3}=2$. Then $\lambda_{y}^{3}=\lambda_{z}^{3}=\lambda_{t}^{3}=3$. By Lemma 3.4 we can get the following matrix.

\begin{tabular}{|c|c|c|c|c|c|c|c|c|c|c|}
\hline$a$ & $a$ & $a$ & $a$ & $*$ & $*$ & $*$ & $*$ & $*$ & $*$ & $*$ \\
\hline$b$ & $c$ & $d$ & $e$ & $b$ & $b$ & $c$ & $c$ & $d$ & $d$ & $e$ \\
\hline$x$ & $y$ & $z$ & $t$ & $z$ & $y$ & $z$ & $t$ & $y$ & $t$ & $x$ \\
\hline$u$ & $v$ & $i$ & $j$ & $*$ & $*$ & $*$ & $*$ & $*$ & $*$ & $v$ \\
\hline
\end{tabular}

Since $d_{2,4}(b, u)=d_{3,4}(z, i)=1$ and (11), we have $a_{4,5}=j$, then $a_{4,7}=u$. So we have a submatrix (rows 1,2,3,4 and columns 2,4,5,7) which is isomorphic to the forbidden configuration $F_{3}$, a contradiction.

2. $\lambda_{x}^{3}=3$.

(i) $d_{2,3}(c, x)=1$, let $a_{3,7}=x$. Since $d_{3,4}(x, u)=d_{3,4}(x, v)=1$, we have $a_{4,7}=j$ by (11). By Lemma 3.2 (forbidden configuration $F_{3}$ ), considering the column set $\{2,3,7\}$ we have $d_{3,4}(z, j)=0$. Then $\lambda_{z}^{3}=2$ and $d_{3,4}(z, u)=1$ by (1). Then we have the following submatrix.

\begin{tabular}{|c|c|c|c|c|c|c|c|c|c|c|}
\hline$a$ & $a$ & $a$ & $a$ & $*$ & $*$ & $*$ & $*$ & $*$ & $*$ & $*$ \\
\hline$b$ & $c$ & $d$ & $e$ & $b$ & $b$ & $c$ & $c$ & $d$ & $d$ & $e$ \\
\hline$x$ & $y$ & $z$ & $t$ & $*$ & $*$ & $x$ & $z$ & $*$ & $*$ & $x$ \\
\hline$u$ & $v$ & $i$ & $j$ & $*$ & $*$ & $j$ & $u$ & $*$ & $*$ & $v$ \\
\hline
\end{tabular}

Thus, the column sets $C_{1}=\{3,7\}$ and $C_{2}=\{4,8\}$ are not separable, a contradiction.

(ii) $d_{2,3}(c, x)=0$, let $a_{3,9}=x$. Since $d_{3,4}(x, u)=d_{3,4}(x, v)=1$, so $a_{4,9}=j$ by (1).

\begin{tabular}{|c|c|c|c|c|c|c|c|c|c|c|}
\hline$a$ & $a$ & $a$ & $a$ & $*$ & $*$ & $*$ & $*$ & $*$ & $*$ & $*$ \\
\hline$b$ & $c$ & $d$ & $e$ & $b$ & $b$ & $c$ & $c$ & $d$ & $d$ & $e$ \\
\hline$x$ & $y$ & $z$ & $t$ & $*$ & $*$ & $*$ & $*$ & $x$ & $*$ & $x$ \\
\hline$u$ & $v$ & $i$ & $j$ & $*$ & $*$ & $*$ & $*$ & $j$ & $*$ & $v$ \\
\hline
\end{tabular}


Thus, the column sets $C_{1}=\{2,9\}$ and $C_{2}=\{3,11\}$ are not separable, a contradiction.

Lemma 3.7 If $A$ is a representation matrix of an $\operatorname{SHF}(4 ; 11,4,\{2,2\})$ and each row of $A$ is isomorphic to $R_{2}$, then there exists a submatrix $B$ satisfying $\lambda_{e}^{2}=\lambda_{f}^{2}=\lambda_{g}^{2}=3$ and $\lambda_{x}^{3}=\lambda_{y}^{3}=\lambda_{z}^{3}=3$.

$$
B=\begin{array}{|l|l|l|}
\hline a & a & a \\
\hline e & f & g \\
\hline x & y & z \\
\hline
\end{array}
$$

Proof: Suppose that $A$ does not contain a submatrix isomorphic to $B$. Then we show that $A$ is not a representation matrix of an $\operatorname{SHF}(4 ; 11,4,\{2,2\})$. Since each row of $A$ is isomorphic to $R_{2}$, we have $\lambda_{d}^{1}=\lambda_{h}^{2}=\lambda_{t}^{3}=\lambda_{j}^{4}=2$. By Lemma 3.1, we have the following submatrix, where $C, D$ and $E$ are $3 \times 3$ matrices, and $F$ is a $3 \times 2$ matrix.

\begin{tabular}{|l|l|l|l|l|l|l|l|l|l|l|}
\hline$a$ & $a$ & $a$ & $b$ & $b$ & $b$ & $c$ & $c$ & $c$ & $d$ & $d$ \\
\hline \multicolumn{3}{|c|}{$\mathrm{C}$} & \multicolumn{3}{|c|}{$\mathrm{D}$} & \multicolumn{3}{|c|}{$\mathrm{E}$} & \multicolumn{2}{|c|}{$\mathrm{F}$} \\
\hline
\end{tabular}

If there is not element in $\{h, t, j\}$ satisfying $d_{1, k}(a, g)=1$, then the submatrix (row set $\{1,2,3\}$ and column set $\{1,2,3\})$ is isomorphic to $B$; If there exists exactly one element $g$ in $\{h, t, j\}$ satisfying $d_{1, k}(a, g)=1$, then the submatrix (row set $\{1,2,3,4\} \backslash\{k\}$ and column set $\{1,2,3\}$ ) is isomorphic to $B$. So the matrix $C$ contains at least two elements in $\{h, t, j\}$. Similarly, the matrices $D$ and $E$ also contain at least two elements in $\{h, t, j\}$, respectively. Since $\lambda_{h}^{2}=\lambda_{t}^{3}=\lambda_{j}^{4}=2$, each of matrices of $C, D$ and $E$ contains two elements of $\{h, t, j\}$. So the matrix $F$ does not contain any one element of $\{h, t, j\}$. Thus, for any two elements $g, g_{1}$ in $\{d, h, t, j\}, d_{k_{1}, k_{2}}\left(g, g_{1}\right)=0,\left\{k_{1}, k_{2}\right\} \subset\{1,2,3,4\}$. By Lemma 3.1, we have the following submatrix.

\begin{tabular}{|c|c|c|c|c|c|c|c|c|c|c|}
\hline$d$ & $d$ & $*$ & $*$ & $*$ & $*$ & $*$ & $*$ & $a$ & $b$ & $c$ \\
\hline$*$ & $*$ & $h$ & $h$ & $*$ & $*$ & $*$ & $*$ & $e$ & $f$ & $h$ \\
\hline$x$ & $y$ & $x$ & $z$ & $t$ & $t$ & $*$ & $*$ & $x$ & $y$ & $z$ \\
\hline$v$ & $*$ & $i$ & $*$ & $*$ & $*$ & $j$ & $j$ & $u$ & $v$ & $i$ \\
\hline
\end{tabular}

Since $a_{4,2}$ and $a_{4,4}$ are in the set $\{u, v, i\}$, we have $\left(a_{4,2}, a_{4,4}\right) \in\{(u, u),(i, u),(u, v)\}$. If $\left(a_{4,2}, a_{4,4}\right)=$ $(u, u)$, then the column sets $C_{1}=\{1,4\}$ and $C_{2}=\{2,3\}$ are not separable, a contradiction; If $\left(a_{4,2}, a_{4,4}\right)=(i, u)$, then $\left(a_{1,3}, a_{1,4}\right)=(b, b)$ by Lemma 3.4, a contradiction; If $\left(a_{4,2}, a_{4,4}\right)=(u, v)$, then $\left(a_{2,1}, a_{2,2}\right)=(e, e)$ by Lemma 3.4, a contradiction.

Lemma 3.8 There is no $\operatorname{SHF}(4 ; 11,4,\{2,2\})$ with all 4 rows isomorphic to $R_{2}$.

Proof: Let $A$ is a representation matrix of an $\operatorname{SHF}(4 ; 11,4,\{2,2\})$. Assume that every row of $A$ is isomorphic to $R_{2}$. By Lemma 3.7, we suppose the first three rows and columns is a submatrix which is isomorphic to $B$ satisfying $\lambda_{e}^{2}=\lambda_{f}^{2}=\lambda_{g}^{2}=3$ and $\lambda_{x}^{3}=\lambda_{y}^{3}=\lambda_{z}^{3}=3$. Suppose the fourth 
elements in rows two and three are $h$ and $t$ respectively. Then $\lambda_{h}^{2}=\lambda_{t}^{3}=2$. We distinguish two cases.

1. $d_{2,3}(h, t)=0$. Without loss of generality, let $d_{2,3}(e, t)=d_{2,3}(f, t)=1$.

(i) $a_{3,5}=a_{3,7}$. Since $d_{2,3}(e, x)=d_{2,3}(f, y)=1$, we have $a_{3,5}=a_{3,7}=z$. So we have the following submatrix.

\begin{tabular}{|c|c|c|c|c|c|c|c|c|c|c|}
\hline$a$ & $a$ & $a$ & $*$ & $*$ & $*$ & $*$ & $*$ & $*$ & $*$ & $*$ \\
\hline$e$ & $f$ & $g$ & $e$ & $e$ & $f$ & $f$ & $g$ & $g$ & $h$ & $h$ \\
\hline$x$ & $y$ & $z$ & $t$ & $z$ & $t$ & $z$ & $x$ & $y$ & $x$ & $y$ \\
\hline$u$ & $v$ & $i$ & $*$ & $*$ & $*$ & $*$ & $*$ & $*$ & $*$ & $*$ \\
\hline
\end{tabular}

By Lemma 3.4, we have $i \notin\left\{a_{4,5}, a_{4,7}, a_{4,8}, a_{4,9}\right\}$. By Lemma 3.2(forbidden configuration $F_{3}$ ), we have $i \notin\left\{a_{4,4}, a_{4,6}, a_{4,10}, a_{4,11}\right\}$, otherwise, we have 4 submatrices (row set $\{1,2,3,4\}$, and column sets $\{2,3,4,6\},\{1,3,4,6\},\{2,3,10,11\},\{1,3,10,11\}$ respectively) which are all isomorphic to $F_{3}$. So we have $\lambda_{i}^{4}=1$, a contradiction.

(ii) $a_{3,5} \neq a_{3,7}$. We distinguish three cases, $\left(a_{3,5}, a_{3,7}\right)=(y, x),(y, z)$, and $(z, x)$. If $\left(a_{3,5}, a_{3,7}\right)=$ $(y, x)$, then $\left(a_{3,10}, a_{3,11}\right)=(z, z)$, a contradiction. $\left(a_{3,5}, a_{3,7}\right)=(y, z)$ and $\left(a_{3,5}, a_{3,7}\right)=(z, x)$ are isomorphic. So let $\left(a_{3,5}, a_{3,7}\right)=(z, x)$.

\begin{tabular}{|c|c|c|c|c|c|c|c|c|c|c|}
\hline$a$ & $a$ & $a$ & $*$ & $*$ & $*$ & $*$ & $*$ & $*$ & $*$ & $*$ \\
\hline$e$ & $f$ & $g$ & $e$ & $e$ & $f$ & $f$ & $g$ & $g$ & $h$ & $h$ \\
\hline$x$ & $y$ & $z$ & $t$ & $z$ & $t$ & $x$ & $x$ & $y$ & $y$ & $z$ \\
\hline$u$ & $v$ & $i$ & $*$ & $*$ & $*$ & $*$ & $*$ & $*$ & $*$ & $*$ \\
\hline
\end{tabular}

By Lemmas 3.2 and 3.4 the possible elements of each position in the last row are listed as below.

\begin{tabular}{|c|c|c|c|c|c|c|c|c|}
\hline$a_{4, k}$ & $a_{4,4}$ & $a_{4,5}$ & $a_{4,6}$ & $a_{4,7}$ & $a_{4,8}$ & $a_{4,9}$ & $a_{4,10}$ & $a_{4,11}$ \\
\hline possible elements & $v, j$ & $v, j$ & $u, j$ & $i, j$ & $v, j$ & $u, j$ & $i, j$ & $u, j$ \\
\hline
\end{tabular}

If $a_{4,4}=v$, by Lemma 3.4, we have $a_{4,5}=j$, then $a_{4,11}=u$. By Lemma 3.2 (forbidden configuration $F_{3}$ ), considering the column set $\{1,2,5\}$, we have $d_{3,4}(y, j)=0$, then $a_{4,9}=u$. So $a_{4,6}=j$ by $\lambda_{u}^{4}=3$. By Lemma 3.2 (forbidden configuration $F_{3}$ ), considering the column set $\{2,3,6\}$, we have $d_{3,4}(z, j)=0$, a contradiction.

If $a_{4,4}=j$, by Lemma 3.4 we have $a_{4,5}=v$ and $a_{4,6}=u$. By Lemma 3.2 (forbidden configuration $\left.F_{3}\right)$, considering the column sets $\{1,2,4\}$ and $\{1,3,4\}$, we have $d_{3,4}(y, j)=d_{34}(z, j)=0$, so $a_{4,9}=a_{4,11}=u$. Thus, $\lambda_{u}^{4}=4$, a contradiction.

2. $d_{2,3}(h, t)=1$. Without loss of generality, let $d_{2,3}(g, t)=1$.

\begin{tabular}{|c|c|c|c|c|c|c|c|c|c|c|}
\hline$a$ & $a$ & $a$ & $*$ & $*$ & $*$ & $*$ & $*$ & $*$ & $*$ & $*$ \\
\hline$e$ & $f$ & $g$ & $e$ & $e$ & $f$ & $f$ & $g$ & $g$ & $h$ & $h$ \\
\hline$x$ & $y$ & $z$ & $y$ & $z$ & $x$ & $z$ & $x$ & $t$ & $t$ & $y$ \\
\hline$u$ & $v$ & $i$ & $*$ & $*$ & $*$ & $*$ & $*$ & $*$ & $*$ & $*$ \\
\hline
\end{tabular}


Similarly, we have the following table.

\begin{tabular}{|c|c|c|c|c|c|c|c|c|}
\hline$a_{4, k}$ & $a_{4,4}$ & $a_{4,5}$ & $a_{4,6}$ & $a_{4,7}$ & $a_{4,8}$ & $a_{4,9}$ & $a_{4,10}$ & $a_{4,11}$ \\
\hline possible elements & $i, j$ & $v, j$ & $i, j$ & $u, j$ & $v, j$ & $u, v, j$ & $j$ & $u, j$ \\
\hline
\end{tabular}

By Lemma 3.4, we have $a_{4,9}=u$ and $a_{4,11}=u$. Then $\lambda_{u}^{4}=3$, and $a_{4,7}=j$. By Lemma 3.4, we have $a_{4,5}=v$ and $a_{4,6}=i$.

\begin{tabular}{|c|c|c|c|c|c|c|c|c|c|c|}
\hline$a$ & $a$ & $a$ & $*$ & $*$ & $*$ & $*$ & $*$ & $*$ & $*$ & $*$ \\
\hline$e$ & $f$ & $g$ & $e$ & $e$ & $f$ & $f$ & $g$ & $g$ & $h$ & $h$ \\
\hline$x$ & $y$ & $z$ & $y$ & $z$ & $x$ & $z$ & $x$ & $t$ & $t$ & $y$ \\
\hline$u$ & $v$ & $i$ & $*$ & $v$ & $i$ & $j$ & $*$ & $u$ & $j$ & $u$ \\
\hline
\end{tabular}

Now, we have a submatrix (rows $1,2,3,4$ and columns $2,3,10,11$ ) which is isomorphic to $F_{3}$, a contradiction.

Theorem 3.9 There exists an optimal $\operatorname{SHF}(4 ; 10,4,\{2,2\})$.

Proof: It's obvious that Lemma 3.8 contradicts with Lemma 3.6. So if there is an $\operatorname{SHF}(4 ; n, 4,\{2,2\})$, then $n \leq 10$. Next, we give a representation matrix of an $\operatorname{SHF}(4 ; 10,4,\{2,2\})$ in the following.

\begin{tabular}{|l|l|l|l|l|l|l|l|l|l|}
\hline 1 & 1 & 1 & 2 & 2 & 2 & 3 & 3 & 3 & 4 \\
\hline 1 & 2 & 3 & 1 & 2 & 3 & 1 & 2 & 3 & 4 \\
\hline 1 & 2 & 3 & 2 & 3 & 1 & 3 & 1 & 2 & 4 \\
\hline 1 & 2 & 3 & 3 & 1 & 2 & 2 & 3 & 1 & 4 \\
\hline
\end{tabular}

Thus, there exists an optimal $\operatorname{SHF}(4 ; 10,4,\{2,2\})$.

Theorem 3.10 If there exists an $\operatorname{SHF}(4 ; n, m,\{2,2\})$ with $m \geq 4$, then $n \leq(m-1)^{2}+1$.

Proof: If $m=4$, the conclusion follows by Theorem [3.9. Now assume that $m>4$. Let $A$ be a representation matrix of an $\operatorname{SHF}(4 ; n, m,\{2,2\})$. Now, we consider the following two cases.

1. There is a pair of elements $x$ and $y$ in the $i$-th row and the $j$-th row respectively such that $d_{i, j}(x, y)>1$. Then we have $n \leq(m-1)^{2}+1$ by Lemma 3.3 .

2. $d_{i, j}(x, y) \leq 1$ for any admissible elements $x, y$ and parameters $i, j$. Then we have $\lambda_{\max } \leq m$. Assume, for a contradiction that $n=(m-1)^{2}+2$. By the pigeonhole principle, there is an element $t_{i}$ such that $\lambda_{t_{i}}^{i} \geq\left\lceil\frac{n}{m}\right\rceil=m-1$ for $1 \leq i \leq 4$.

(i) $\lambda_{\max }=m$. Assume that there are two elements $a$ and $k$ in different rows (without loss of generality, in the first two rows) such that $\lambda_{a}^{1}=\lambda_{k}^{2}=m$. Then there is a submatrix of $A$ as below. 


\begin{tabular}{|c|c|l|c|c|c|c|c|c|}
\hline$a$ & $a$ & $\cdots$ & $a$ & $a$ & $*$ & $*$ & $\cdots$ & $*$ \\
\hline$*$ & $*$ & $\cdots$ & $*$ & $k$ & $k$ & $k$ & $\cdots$ & $k$ \\
\hline$x_{1}$ & $x_{2}$ & $\cdots$ & $x_{m-1}$ & $x_{m}$ & $y_{1}$ & $y_{2}$ & $\cdots$ & $y_{m-1}$ \\
\hline$u_{1}$ & $u_{2}$ & $\cdots$ & $u_{m-1}$ & $u_{m}$ & $v_{1}$ & $v_{2}$ & $\cdots$ & $v_{m-1}$ \\
\hline
\end{tabular}

By Lemma 3.2 (forbidden configuration $\left.F_{3}\right), d_{3,4}\left(x_{i}, v_{j}\right)=0$ and $d_{4,3}\left(u_{i}, y_{j}\right)=0$ for any $u_{i} \neq v_{j}$, $x_{i} \neq y_{j}, 1 \leq i, j \leq m-1$. So, there are at least $(m-1) \times(m-3)$ distinct pairs of elements $s$ and $t$ such that $d_{3,4}(s, t)=0$. Thus, $(m-1) \times(m-3)+n \leq m^{2}$, ie. $(m-1) \times(m-3)+(m-1)^{2}+2 \leq m^{2}$, so we have $m \leq 4$. It contradicts $m>4$.

So we may assume that there is exactly one row (without loss of generality, the first row) containing an element $a$ such that $\lambda_{a}^{1}=m$.

\begin{tabular}{|c|c|l|c|c|c|c|c|c|}
\hline$a$ & $a$ & $\cdots$ & $a$ & $a$ & $*$ & $*$ & $\cdots$ & $*$ \\
\hline$*$ & $*$ & $\cdots$ & $*$ & $k$ & $k$ & $k$ & $\cdots$ & $k$ \\
\hline$x_{1}$ & $x_{2}$ & $\cdots$ & $x_{m-1}$ & $x_{m}$ & $y_{1}$ & $y_{2}$ & $\cdots$ & $y_{m-2}$ \\
\hline$u_{1}$ & $u_{2}$ & $\cdots$ & $u_{m-1}$ & $u_{m}$ & $v_{1}$ & $v_{2}$ & $\cdots$ & $v_{m-2}$ \\
\hline
\end{tabular}

Similarly, we can obtain $(m-2) \times(m-3)$ distinct pairs of elements $s$ and $t$ such that $d_{3,4}(s, t)=0$. Since $\lambda_{x_{m}}^{3} \leq m-1$ and $\lambda_{u_{m}}^{4} \leq m-1$, we know that there are two elements $w$ in row three and $z$ in row four such that $d_{3,4}\left(x_{m}, z\right)=d_{3,4}\left(w, u_{m}\right)=0$. Thus, $(m-2) \times(m-3)+2+n \leq m^{2}$ contradicts $m>4$.

(ii) $\lambda_{\max }=m-1$. Then there exist two elements $a$ and $k$ in the first two rows such that $\lambda_{a}^{1}=\lambda_{k}^{2}=m-1$. Suppose there is a submatrix of $A$ as blew.

\begin{tabular}{|c|c|c|c|c|c|c|c|c|}
\hline$a$ & $a$ & $\cdots$ & $a$ & $a$ & $*$ & $*$ & $\cdots$ & $*$ \\
\hline$*$ & $*$ & $\cdots$ & $*$ & $k$ & $k$ & $k$ & $\cdots$ & $k$ \\
\hline$x_{1}$ & $x_{2}$ & $\cdots$ & $x_{m-2}$ & $x_{m-1}$ & $y_{1}$ & $y_{2}$ & $\cdots$ & $y_{m-2}$ \\
\hline$u_{1}$ & $u_{2}$ & $\cdots$ & $u_{m-2}$ & $u_{m-1}$ & $v_{1}$ & $v_{2}$ & $\cdots$ & $v_{m-2}$ \\
\hline
\end{tabular}

Similarly, there are at least $(m-2) \times(m-4)+2$ distinct pairs of elements $s$ and $t$ such that $d_{3,4}(s, t)=0$. Thus, $(m-2) \times(m-4)+2+n \leq m^{2}$. So we have $m \leq 5$. If $m>5$, we have a contradiction. Now we assume that $m=5$. Then we have the following submatrix of $A$.

\begin{tabular}{|c|c|c|c|c|c|c|}
\hline$a$ & $a$ & $a$ & $a$ & $*$ & $*$ & $*$ \\
\hline$*$ & $*$ & $*$ & $k$ & $k$ & $k$ & $k$ \\
\hline$x_{1}$ & $x_{2}$ & $x_{3}$ & $x_{4}$ & $y_{1}$ & $y_{2}$ & $y_{3}$ \\
\hline$u_{1}$ & $u_{2}$ & $u_{3}$ & $u_{4}$ & $v_{1}$ & $v_{2}$ & $v_{3}$ \\
\hline
\end{tabular}

Let $X_{1}=\left\{x_{1}, x_{2}, x_{3}\right\}, Y_{1}=\left\{y_{1}, y_{2}, y_{3}\right\}, U_{1}=\left\{u_{1}, u_{2}, u_{3}\right\}$ and $V_{1}=\left\{v_{1}, v_{2}, v_{3}\right\}$. Since $d_{i, j}(x, y) \leq 1$, we have $\left|\left\{x_{1}, x_{2}, x_{3}, x_{4}\right\}\right|=\left|\left\{x_{4}, y_{1}, y_{2}, y_{3}\right\}\right|=4$. Then by $m=5$ we have $2 \leq\left|X_{1} \cap Y_{1}\right| \leq 3$. Similarly, we can get $2 \leq\left|U_{1} \cap V_{1}\right| \leq 3$. Now we continue to distinguish the following 2 cases.

(a) $\left|X_{1} \cap Y_{1}\right|=3$ and $\left|U_{1} \cap V_{1}\right|=3$. Thus, $X_{1}=Y_{1}$ and $U_{1}=V_{1}$. By Lemma 3.1 we only need to consider the following two submatrices. 


\begin{tabular}{|c|c|c|c|c|c|c|}
\hline$a$ & $a$ & $a$ & $a$ & $*$ & $*$ & $*$ \\
\hline$*$ & $*$ & $*$ & $k$ & $k$ & $k$ & $k$ \\
\hline$x_{1}$ & $x_{2}$ & $x_{3}$ & $x_{4}$ & $x_{1}$ & $x_{2}$ & $x_{3}$ \\
\hline$u_{1}$ & $u_{2}$ & $u_{3}$ & $u_{4}$ & $u_{2}$ & $u_{3}$ & $u_{1}$ \\
\hline
\end{tabular}

\begin{tabular}{|c|c|c|c|c|c|c|}
\hline$a$ & $a$ & $a$ & $a$ & $*$ & $*$ & $*$ \\
\hline$*$ & $*$ & $*$ & $k$ & $k$ & $k$ & $k$ \\
\hline$x_{1}$ & $x_{2}$ & $x_{3}$ & $x_{4}$ & $x_{1}$ & $x_{2}$ & $x_{3}$ \\
\hline$u_{1}$ & $u_{2}$ & $u_{3}$ & $u_{4}$ & $u_{3}$ & $u_{1}$ & $u_{2}$ \\
\hline
\end{tabular}

It is easy to check that in the left submatrix column sets $C_{1}=\{1,6\}$ and $C_{2}=\{3,5\}$ are not separable, and in the right submatrix the column sets $C_{1}=\{1,7\}$ and $C_{2}=\{2,5\}$ are not separable, a contradiction.

(b) $\left|X_{1} \cap Y_{1}\right|=2$ and $\left|U_{1} \cap V_{1}\right| \leq 3$. By Lemma 3.1, we may assume that $y_{1}=x_{1}, y_{2}=x_{2}$ and $y_{3}=x_{5}$. So we have the following submatrix.

\begin{tabular}{|c|c|c|c|c|c|c|}
\hline$a$ & $a$ & $a$ & $a$ & $*$ & $*$ & $*$ \\
\hline$*$ & $*$ & $*$ & $k$ & $k$ & $k$ & $k$ \\
\hline$x_{1}$ & $x_{2}$ & $x_{3}$ & $x_{4}$ & $x_{1}$ & $x_{2}$ & $x_{5}$ \\
\hline$u_{1}$ & $u_{2}$ & $u_{3}$ & $u_{4}$ & $v_{1}$ & $v_{2}$ & $v_{3}$ \\
\hline
\end{tabular}

For each $i=1,2$, since $\left|V_{1} \backslash\left\{v_{i}, u_{i}\right\}\right| \geq 1$, we know that there is at least one element $t_{i} \in$ $V_{1} \backslash\left\{v_{i}, u_{i}\right\}$ such that $d_{3,4}\left(x_{i}, t_{i}\right)=0$ by Lemma 3.2 (forbidden configuration $F_{3}$ ).

Similarly, since $\left|V_{1} \backslash\left\{u_{3}\right\}\right| \geq 2$ and $\left|U_{1} \backslash\left\{v_{3}\right\}\right| \geq 2$ we know that there are at least two elements $t_{3}, t_{4} \in V_{1} \backslash\left\{u_{3}\right\}$ and $t_{5}, t_{6} \in U_{1} \backslash\left\{v_{3}\right\}$ such that $d_{3,4}\left(x_{3}, t_{3}\right)=0, d_{3,4}\left(x_{3}, t_{4}\right)=0$ and $d_{3,4}\left(x_{5}, t_{5}\right)=0$, $d_{3,4}\left(x_{5}, t_{6}\right)=0$ respectively.

Since $m=5, \lambda_{x_{4}}^{3} \leq 4$, and $\lambda_{u_{4}}^{4} \leq 4$, we know that there are two elements $w$ in row three and $z$ in row four such that $w \neq x_{4}, z \neq u_{4}$ and $d_{3,4}\left(x_{4}, z\right)=d_{3,4}\left(w, u_{4}\right)=0$.

Since $x_{4} \notin X_{1} \cup Y_{1}$ and $u_{4} \notin U_{1} \cup V_{1}$ we have obtained at least 8 distinct pairs of elements $(s, t)$ such that $d_{3,4}(s, t)=0$. So we have $8+n=8+18=26>25$, a contradiction.

The proof is complete.

Remark 2 : It's easy to see that the bound in Theorem 3.10 is tight for $m=4$ by Theorem 3.9 .

Now we are in the position to prove the main result of this section.

Theorem 3.11 If there exists an $\operatorname{SHF}(2 w ; n, m,\{w, w\})$ with $m \geq 2 w \geq 4$, then $n \leq(m-1)^{2}+1$.

Proof: We use induction on $w$ to prove the theorem.

1. By Theorem 3.10, for $w=2$ this conclusion holds.

2. Assume the conclusion holds for $w=k-1, k \geq 3$. Then, we consider the case $w=$ $k$. Let $m \geq 2 k \geq 6$. Assume, for a contradiction, that $A$ is a representation matrix of an $\operatorname{SHF}\left(2 k ;(m-1)^{2}+2, m,\{k, k\}\right)$, and $\mathcal{C}$ denote the set of all columns of $A$. By removing the first two rows of $A$, we obtain a $(2 k-2) \times\left(m^{2}-2 m+3\right)$ submatrix $B$. By inductive hypothesis, $B$ is not an $\operatorname{SHF}\left(2 k-2 ;(m-1)^{2}+2, m,\{k-1, k-1\}\right)$. Thus, there are two disjoint subsets of columns $C_{1}$ and $C_{2}$ of $B$ with $\left|C_{1}\right|=\left|C_{2}\right|=k-1$ which are not separable. Now, we consider the same 
column sets $C_{1}$ and $C_{2}$ in $A$. Let $D=\mathcal{C} \backslash\left(C_{1} \cup C_{2}\right)$, then $|D|=m^{2}-2 m-2 k+5 \geq m^{2}-3 m+5>m$. Let $Y_{i}, U_{i}$ and $V_{i}(i=1,2)$ be the element sets in which each element appears in the $i$-th row of these columns in $D, C_{1}$ and $C_{2}$ respectively.

If there exist two elements $x \in Y_{1}$ and $y \in Y_{2}$ such that $d_{1,2}(x, y) \geq 2$, then we have two distinct columns $l_{1}$ and $l_{2}$ agreeing in the first two rows. So $C_{1} \cup\left\{l_{1}\right\}$ and $C_{2} \cup\left\{l_{2}\right\}$ are not separated in $A$, a contradiction. Now, assume that $d_{1,2}(x, y) \leq 1$ for all $x \in Y_{1}$ and $y \in Y_{2}$. If $\left\{a_{1, j_{1}}: j_{1} \in C_{1}\right\} \cap\left\{a_{1, j_{2}}: j_{2} \in C_{2}\right\} \neq \emptyset$, then we have two distinct columns $l_{1}$ and $l_{2}$ agreeing in the second row. So $C_{1} \cup\left\{l_{1}\right\}$ and $C_{2} \cup\left\{l_{2}\right\}$ are not separated in $A$, a contradiction. By Lemma 3.1, we only need to consider the case that $\left\{a_{i, j_{1}}: j_{1} \in C_{1}\right\} \cap\left\{a_{i, j_{2}}: j_{2} \in C_{2}\right\}=\emptyset, 1 \leq i \leq 2$, and $d_{1,2}(x, y) \leq 1$ for all $x \in Y_{1}$ and $y \in Y_{2}$. Suppose that $\left|Y_{1}\right| \geq\left|Y_{2}\right|$, we distinguish the following four cases.

(i) $\left|Y_{1}\right| \leq m-1$. Since $\left\lceil\frac{n-2(k-1)}{m-1}\right\rceil=m-1$, by the pigeonhole principle, there exists an element $x \in Y_{1}$ such that $\lambda_{x}^{1} \geq m-1$ in $D$. Since $d_{1,2}(x, y) \leq 1$ for $x \in Y_{1}$ and $y \in Y_{2}$, we have two columns $l_{1}$ and $l_{2}$ in $D$ such that $a_{1, l_{1}}=a_{1, l_{2}}=x$ and $a_{2, l_{1}} \in U_{2} \cup V_{2}$. If $a_{2, l_{1}} \in U_{2}$, then $C_{1} \cup\left\{l_{2}\right\}$ is not separated from $C_{2} \cup\left\{l_{1}\right\}$, a contradiction. Similarly, we have a contradiction for $a_{2, l_{1}} \in V_{2}$.

(ii) $\left|Y_{1}\right|=m$ and $\left|Y_{2}\right|<m-1$. Since $\left\lceil\frac{n-2(k-1)}{m-2}\right\rceil=m$, by the pigeonhole principle, there exists an element $x \in Y_{2}$ such that $\lambda_{x}^{2} \geq m$ in $D$. Then we have two columns $l_{1}$ and $l_{2}$ in $D$ such that $a_{2, l_{1}}=a_{2, l_{2}}=x$ and $a_{1, l_{1}} \in U_{1}$. So $C_{1} \cup\left\{l_{2}\right\}$ is not separated from $C_{2} \cup\left\{l_{1}\right\}$, a contradiction.

(iii) $\left|Y_{1}\right|=m$ and $\left|Y_{2}\right|=m-1$. Since $\left\lceil\frac{n-2(k-1)}{m-1}\right\rceil=m-1$, by the pigeonhole principle, there exists an element $x \in Y_{2}$ such that $\lambda_{x}^{2} \geq m-1$ in $D$. We may have a contradiction by using the similar method in case (i).

(iv) $\left|Y_{1}\right|=\left|Y_{2}\right|=m$. For any column $k_{i} \in C_{i}(i=1,2)$, we have $a_{1, k_{1}} \in Y_{1}, a_{1, k_{2}} \in Y_{1}$, $a_{2, k_{1}} \in Y_{2}$ and $a_{2, k_{2}} \in Y_{2}$. If there exist distinct columns $l_{1}$ and $l_{2}$ in $D$, such that $a_{1, k_{2}}=a_{1, l_{1}}$ and $a_{2, k_{1}}=a_{2, l_{2}}$, we have that $C_{1} \cup\left\{l_{1}\right\}$ is not separated from $C_{2} \cup\left\{l_{2}\right\}$, a contradiction. Otherwise, there exists the unique column $l$ such that $a_{1, k_{2}}=a_{1, l}$, or $a_{2, k_{1}}=a_{2, l}$, so $a_{1, k_{2}}=a_{1, l}$ and $a_{2, k_{1}}=a_{2, l}$. Similarly, we have a unique column $l^{\prime}$ such that $a_{1, k_{1}}=a_{1, l^{\prime}}$ and $a_{2, k_{2}}=a_{2, l^{\prime}}$. Then there are $m-2$ distinct elements in the first row of column set $D \backslash\left\{l, l^{\prime}\right\}$ and $m-2$ distinct elements in the second row of column set $D \backslash\left\{l, l^{\prime}\right\}$. Since $\left\lceil\frac{n-2(k-1)-2}{m-2}\right\rceil=m-1>m-2$, by the pigeon hole principle, there exist two elements $x \in Y_{1}$ and $y \in Y_{2}$ such that $d_{1,2}(x, y)>1$, a contradiction.

The proof is complete.

Remark 3 : The best upper bound for an $\operatorname{SHF}(2 w ; n, m,\{w, w\})$ with $m \geq 2 w \geq 4$ is $n<m^{2}$ [4]. We improve this bound from $n<m^{2}$ to $n \leq(m-1)^{2}+1$.

\section{An improved bound for $\operatorname{SHF}\left(\sum_{i=1}^{t} w_{i} ; n, m,\left\{w_{1}, w_{2}, \ldots, w_{t}\right\}\right)$}

In this section, we shall give a new bound for an $\operatorname{SHF}\left(w_{1}+w_{2} ; n, m,\left\{w_{1}, w_{2}\right\}\right)$ with $w_{2}>w_{1} \geq 2$ and $m \geq w_{1}+w_{2}$. Then we obtain a new bound for $\operatorname{SHF}\left(\sum_{i=1}^{t} w_{i} ; n, m,\left\{w_{1}, w_{2}, \ldots, w_{t}\right\}\right)$. 
Lemma 4.1 Suppose $A$ is a representation matrix of an $\operatorname{SHF}(2+w ; n, m,\{2, w\})$ with $m \geq 2+w$. If there is a pair of elements $a$ and $b$ such that $d_{i, j}(a, b) \geq 2$, then $n<m^{2}-m$.

Proof: Suppose, for a contradiction, that $A$ is a representation matrix of an $\operatorname{SHF}\left(2+w ; m^{2}-\right.$ $m, m,\{2, w\})$ with $m \geq 2+w$, and $d_{12}(a, b) \geq 2$. Let $\mathcal{C}$ denote the set of columns of $A$, and $\mathcal{B}=\left\{(i, j): \lambda_{a_{i, j}}^{i}=1,3 \leq i \leq w+2,1 \leq j \leq 2\right\}$. We distinguish the following 4 cases.

1. $|\mathcal{B}|=0$. Then there are two columns $l_{i}$ and $l_{i}^{\prime}$ of $A$ such that $a_{i, 1}=a_{i, l_{i}}$ and $a_{i, 2}=a_{i, l_{i}^{\prime}}$ for each $3 \leq i \leq w+2$. Let $C_{1}=\{2\} \cup\left\{l_{i}: 3 \leq i \leq w+2\right\}$ and $C_{2}=\{1\} \cup\left\{l_{i}^{\prime}: 3 \leq i \leq w+2\right\}$. If there is a column $h_{1} \notin C_{1}$ or $h_{2} \notin C_{1}$ satisfying $a_{w+1,2}=a_{w+1, h_{1}}$ or $a_{w+2,2}=a_{w+2, h_{2}}$ respectively, then $C_{1} \backslash\left\{l_{w+j}\right\}$ is not separated from $\left\{1, h_{j}\right\}$ for $j=1$ or 2 , a contradiction. Thus, for any column $l$ satisfying $a_{w+1,2}=a_{w+2, l}$ or $a_{w+2,2}=a_{w+2, l}$ we have $l \in C_{1}$. Similarly, if $a_{w+1,1}=a_{w+2, l^{\prime}}$ or $a_{w+2,1}=a_{w+2, l^{\prime}}$, we have $l^{\prime} \in C_{2}$. Let $\mathcal{C}_{1}=\mathcal{C} \backslash\left(C_{1} \cup C_{2}\right)$. By the pigeonhole principle, there is an element $t$ in row $w+2$ such that $\lambda_{t}^{w+2} \geq\left\lceil\frac{n-2 w-2}{m-2}\right\rceil>m-2$. So there exist two columns $l_{1}^{\prime}$ and $l_{2}^{\prime}$ of $\mathcal{C}_{1}$ agreeing in the last two rows. Then $\left\{1, l_{1}^{\prime}\right\}$ and $C_{1} \cup\left\{l_{2}^{\prime}\right\} \backslash\left\{l_{w+1}, l_{w+2}\right\}$ are not separated, a contradiction.

2. $|\mathcal{B}|=1$. By Lemma 3.1. we may assume $\lambda_{a_{w+2,1}}^{w+2}=1, \lambda_{a_{w+2,2}}^{w+2} \geq 2$ and $\lambda_{a_{i, j}}^{i} \geq 2,3 \leq i \leq$ $w+1,1 \leq j \leq 2$. Then there exist a column $l_{i}$ of $A$ such that $a_{i, 1}=a_{i, l_{i}}$ for each $3 \leq i \leq w+1$. Let $C_{1}=\{2\} \cup\left\{l_{i}: 3 \leq i \leq w+1\right\}$. If there exists a column $l$ such that $a_{w+2,2}=a_{w+2, l}$ with $l \notin C_{1}$, then let $C_{2}=\{1, l\}$, so we have $C_{1}$ is not separated from $C_{2}$, a contradiction. Thus, for any column $l$ satisfying $a_{w+2,2}=a_{w+2, l}$, we have $l \in C_{1}$. Let $\mathcal{C}_{1}=\mathcal{C} \backslash\left(C_{1} \cup\{1\}\right)$. By the pigeonhole principle, there is an element $t$ in row $w+2$ such that $\lambda_{t}^{w+2} \geq\left\lceil\frac{n-w-1}{m-2}\right\rceil>m$. So there exist two columns $l_{1}$ and $l_{2}$ of $\mathcal{C}_{1}$ agreeing in the last two rows. Then $\left\{1, l_{1}\right\}$ and $C_{1} \cup\left\{l_{2}\right\} \backslash\left\{l_{w+1}\right\}$ are not separated, a contradiction.

3. $|\mathcal{B}|=2$. Let $\lambda_{x}^{i_{1}}=\lambda_{y}^{i_{2}}=1$.

(i) $i_{1}=i_{2}$. By Lemma 3.1, we may assume $i_{1}=i_{2}=w+2$. Then there exist a column $l_{i}$ of $A$ such that $a_{i, 1}=a_{i, l_{i}}$ for each $3 \leq i \leq w$. Let $C_{1}=\{2\} \cup\left\{l_{i}: 3 \leq i \leq w\right\}$. Let $\mathcal{C}_{1}=\mathcal{C} \backslash\left(C_{1} \cup\{1\}\right)$. By the pigeonhole principle, there is an element $t$ in row $w+2$ such that $\lambda_{t}^{w+2} \geq\left\lceil\frac{n-w}{m-2}\right\rceil>m$. So there exist two columns $l_{1}$ and $l_{2}$ of $\mathcal{C}_{1}$ agreeing in the last two rows. Then $\left\{1, l_{1}\right\}$ and $C_{1} \cup\left\{l_{2}\right\}$ are not separated, a contradiction.

(ii) $i_{1} \neq i_{2}$. By Lemma 3.1, we may assume $i_{1}=w+1$ and $i_{2}=w+2$. Then there exist a column $l_{i}$ of $A$ such that $a_{i, 1}=a_{i, l_{i}}$ for each $3 \leq i \leq w$. Let $C_{1}=\{2\} \cup\left\{l_{i}: 3 \leq i \leq w\right\}$. Let $\mathcal{C}_{1}=\mathcal{C} \backslash\left(C_{1} \cup\{1\}\right)$. By the pigeonhole principle, there is an element $t$ in row $w+2$ such that $\lambda_{t}^{w+2} \geq\left\lceil\frac{n-w}{m-1}\right\rceil>m-1$. So we have two columns $l_{1}$ and $l_{2}$ agreeing in the last two rows, and $\left\{1, l_{1}\right\}$ and $C_{1} \cup\left\{l_{2}\right\}$ are not separable, a contradiction.

4. $|\mathcal{B}| \geq 3$. Let $3 \leq k \leq w$. By Lemma 3.1, we may assume that $\lambda_{a_{i, j}}^{i}>1$ when $3 \leq i \leq k$ and $1 \in\left\{\lambda_{a_{i, 1}}^{i}, \lambda_{a_{i, 2}}^{i}\right\}$ when $k+1 \leq i \leq w+2$. Then there exist a column $l_{i}$ of $A$ such that $a_{i, 1}=a_{i, l_{i}}$ for each $3 \leq i \leq k$. Let $C_{1}=\{2\} \cup\left\{l_{i}: 2<i \leq k\right\}$, and let $\mathcal{C}_{1}=\mathcal{C} \backslash\left(C_{1} \cup\{1\}\right)$. Then $\left|\mathcal{C}_{1}\right|=m^{2}-m-k>(m-1)^{2}$. From [4] (Theorem 10), we have two columns sets $C_{2}$ and $C_{3}$ satisfying $\left|C_{2}\right|=1,\left|C_{3}\right|=w+1-k$, and $C_{2}$ and $C_{3}$ are not separable in the rows from $k+1$ to 
$w+2$. Thus, we have $C_{1} \cup C_{3}$ is not separated from $C_{2} \cup\{1\}$. The proof is complete.

Theorem 4.2 Suppose $A$ is a representation matrix of an $\operatorname{SHF}(2+w ; n, m,\{2, w\})$ with $w \geq 2$ and $m \geq 2+w$, then $n<m^{2}-m$.

Proof: We use induction on $w$ to prove the theorem.

1. By Theorem 3.10, for $w=2$ this case satisfies.

2. Assume the conclusion holds for $w=k-1, k \geq 3$. Suppose, for a contradiction, that an $\operatorname{SHF}\left(k+2 ; m^{2}-m+3, m,\{2, k\}\right)$ exists with $A$ as the representation matrix. Let $\mathcal{C}$ denote the set of columns of $A$. By removing the first row of $A$, we obtain a $(k+1) \times\left(m^{2}-m+3\right)$ submatrix $B$. By inductive hypothesis, there are two sets of columns $C_{1}$ and $C_{2}$ in $B$ with $\left|C_{1}\right|=2$ and $\left|C_{2}\right|=k-1$ which are not separable. Now, we consider the same column sets $C_{1}$ and $C_{2}$ in $A$. Let $\mathcal{C}_{1}=\mathcal{C} \backslash C_{1}$. If there exist two columns $l_{1}$ and $l_{2}$ satisfying $l_{1} \in \mathcal{C}_{1}$ and $l_{2} \in C_{1}$ such that $a_{1, l_{1}}=a_{1, l_{2}}$, then $C_{1}$ is not separated from $C_{2} \cup\left\{l_{1}\right\}$, a contradiction; Now we have that for any two columns $l_{1} \in \mathcal{C}_{1}$ and $l_{2} \in C_{1}$ satisfy $a_{1, l_{1}} \neq a_{1, l_{2}}$. Thus, $\left|\left\{a_{1, i}: i \in \mathcal{C}_{1}\right\}\right| \leq m-1$. Similarly, we have $\left|\left\{a_{2, i}: i \in \mathcal{C}_{1}\right\}\right| \leq m-1$. By the pigeonhole principle, there is an element $t$ in the first row such that $\lambda_{t}^{1} \geq\left\lceil\frac{n-2}{m-1}\right\rceil>m-1$. So we have $d_{1,2}(t, z) \geq 2$ in $\mathcal{C}_{1}$. It contradicts Lemma 4.1. The proof is complete.

We use Theorem 4.2 as the inductive hypothesis to prove the following theorem. The proof method is similar to the Theorem 3.11 .

Theorem 4.3 If there exists an $\operatorname{SHF}\left(w_{1}+w_{2} ; n, m,\left\{w_{1}, w_{2}\right\}\right)$ with $2 \leq w_{1}<w_{2}$ and $m \geq w_{1}+w_{2}$, then $n<m^{2}-m$.

Remark 4 : The best upper bound for an $\operatorname{SHF}\left(w_{1}+w_{2} ; n, m,\left\{w_{1}, w_{2}\right\}\right)$ with $m \geq w_{1}+w_{2}$ is $n \leq m^{2}$ [4]. When $w_{1}=1$ and $w_{2} \geq 2$, this bound is tight. When $w_{2}>w_{1} \geq 2$, we improve this bound from $n \leq m^{2}$ to $n<m^{2}-m$.

Now, we obtain the mainly conclusion of SHFs in the following.

Theorem 4.4 Suppose there exists an $\operatorname{SHF}\left(u ; n, m,\left\{w_{1}, w_{2}, \ldots, w_{t}\right\}\right)$ with $u=\sum_{i=1}^{t} w_{i}$. If $m \geq u$ and $\left\{w_{1}, w_{2}, \ldots, w_{t}\right\} \notin\{\{1,1,1\},\{1, w\}\}$, then $n<m^{2}-m$.

Proof: If $t=2$, since $\left\{w_{1}, w_{2},\right\} \neq\{1, w\}$ we have $n<m^{2}-m$ by Theorems 3.11 and 4.3, Now we assume $t \geq 3$. By the definition of an $\operatorname{SHF}$, we know that an $\operatorname{SHF}\left(u ; n, m,\left\{w_{1}, w_{2}, \ldots, w_{t}\right\}\right)$ is also an $\operatorname{SHF}\left(u ; n, m,\left\{w, w^{\prime}\right\}\right)$ with $w=w_{1}+w_{2}$ and $w^{\prime}=\sum_{i=3}^{t} w_{i}$. Since $\left\{w_{1}, w_{2}, \ldots, w_{t}\right\} \neq\{1,1,1\}$, we have $w \geq 2$ and $w^{\prime} \geq 2$. By Theorems 3.11 and 4.3, we have $n<m^{2}-m$.

Remark 5: The best upper bound for an $\operatorname{SHF}\left(N ; n, m,\left\{w_{1}, w_{2}, \ldots, w_{t}\right\}\right)$ is $n \leq r m^{\left\lceil\frac{N}{u-1}\right\rceil}+(u-$ r) $m^{\left\lfloor\frac{N}{u-1}\right\rfloor}$ where $u=\sum_{i=1}^{t} w_{i}, 1 \leq r \leq u-1$ and $N \equiv r(\bmod u-1)$ [23]. When $N=u$, we improve this bound from $n \leq m^{2}+(u-1) m$ to $n<m^{2}-m$. 


\section{References}

[1] N. Alon and U. Stav, New bounds on parent-identifying codes: the case of multiple parents, Combin. Probab. Comput. 13 (2004), 795-807.

[2] M. Atici, S. S. Magliveras, D. R. Stinson, and W. D. Wei, Some recursive constructions for perfect hash families, J. Combin. Des. 4 (1996), 353-363.

[3] M. Bazrafshan and T. Trung, Bounds for separating hash families, J. Combin. Theory Ser. A 118 (2011), 1129-1135.

[4] M. Bazrafshan and T. Trung, Improved bounds for separating hash families, Des. Codes Cryptogr. 69 (2013), 369-382.

[5] S. R. Blackburn, Frameproof codes, SIAM J. Discrete Math. 16 (2003), 499-510.

[6] S. R. Blackburn, T. Etzion, D. R. Stinson, and G. M. Zaverucha, A bounds on the size of separating hash families, J. Combin. Theory Ser. A 115 (2008), 1246-1256.

[7] D. Boneh and J. Shaw, Collusion-free fingerprinting for digital data, IEEE Trans. Inf. Theory. 44 (1998), 1897-1905.

[8] C. J. Colbourn and J. H. Dinitz, Handbook of Combinatorial Designs, 2nd Ed Chapman \& Hall/CRC,(2007).

[9] Y. Caro and R. Yuster, Covering graphs: The covering problem solved, J. Combin. Theory Ser. A 83 (1998), 273-282.

[10] R. Fuji-Hara, Perfect hash families of strength three with three rows from varieties on finite projective geometries, Des. Codes Cryptogr. 77 (2015), 351-356.

[11] F. Gao and G. Ge, New Bounds on Separable Codes for Multimedia Fingerprinting, IEEE Trans. Inf. Theory. 60 (2014), 5257-5262.

[12] G. Ge, C. Shangguan, and X. Wang, Some intriguing upper bounds for separating hash families, http://arXiv:1707.01758v1, 2017.

[13] H. D. O. F. Gronau, R. C. Mullin, and C. Pietsch, The closure of all subsets of $\{3,4, \ldots, 10\}$ which include 3, Ars Combin. 41 (1995) 129-161.

[14] C. Guo, D. R. Stinson, and T. Trung, On tight bounds for binary frameproof codes, Des. Codes Cryptogr. 77 (2015), 301-319.

[15] C. Guo and D. R. Stinson, A tight bound on the size of certain separating hash families, Australas. J. Combin. 67 (2017), 294-303.

[16] H.Hanani, On quadruple systems, Canad. J. Math. 12 (1960), 12:145-157.

[17] H.Hanani, Balanced incomplete block designs and related designs, Discrete Math. 11 (1975), 255-369.

[18] D. R.Hughes, On t-designs and groups, Amer. J. Math. 87 (1965), 761-778.

[19] P. C. Li, G. H. J. van Rees, and R. Wei, Constructions of 2-cover-free families and related separating hash families, J. Combin. Des. 14 (2006), 423-440.

[20] L. Liu and H. Shen, Explicit constructions of separating hash families from algebraic curves over finite fields, Des. Codes Cryptogr. 41 (2006), 221-233.

[21] V. Roödl, On a packing and covering problem, Eur. J. Combin. 6 (1985), 69-78.

[22] P. Sarkar and D. R. Stinson, Frameproof and IPP codes, Lecture Notes in Comput Sci. 2247 (2001), 117-126. 
[23] C. Shangguan and G. Ge, Separating hash families: A Johnson-type bound and new constructions, SIAM J. Discrete Math. 30 (2016), 2243-2264.

[24] C. Shangguan, X. Wang, G. Ge, and Y. Miao, New bounds for frameproof codes, http:// arXiv:1411.5782v1, 2014.

[25] J. N. Staddon, D. R. Stinson, and R. Wei, Combinatorial properties of frameproof and traceability codes, IEEE Trans. Inf. Theory. 47 (2001), 1042-1049.

[26] D. R. Stinson, T. Trung, and R.Wei, Secure frameproof codes, key distribution patterns, group testing algorithms and related structures, J. Statist. Plan. Inference. 86 (2000), 595-617.

[27] D. R. Stinson, R. Wei, and K. Chen, On generalized separating hash families, J. Combin. Theory Ser. A 115 (2008), 105-120.

[28] R. A. Walker II and C. J. Colbourn, Perfect hash families: constructions and existence, J. Math. Cryptogr. 1 (2007), 125-150. 